\title{
Original
}

\section{Ultrasound Elastographic Assessment of the Medial Collateral Ligament in the Elbow Joints of Baseball Players}

\author{
Kazusa WADA ${ }^{* 1)}$, Mikihiko WatAnABE ${ }^{2)}$ Kazunari Tomita $^{3)}$, \\ Motoaki TAKASHIMA $^{1)}$ and Katsunori INAGAKI $^{1)}$
}

\begin{abstract}
We investigated the elasticity of the medial collateral ligament (MCL) in baseball players using ultrasound elastography (EG). We examined 72 elbows in 36 male baseball players (including 12 junior high students, 12 high school students, and 12 university students) with no history of pitching injuries. We used the Hitachi Medical Corporation HI VISION Avius, an acoustic coupler for quantification, as well as real-time tissue EG, which was applied to subjects in the supine position. Pressure was applied manually with the subject's forearm in supination, elbow joint at $30^{\circ}$ of flexion, and shoulder joint at $90^{\circ}$ abduction. The strain ratio (SR) of the coupler and MCL was used for evaluation. SR increased with age $(P<0.05)$ in both arms, and the SR in the university student group was higher than that in the other groups $(P<0.05)$. In addition, the throwing side exhibited significantly lower SR values compared to the non-throwing side $(P<0.05)$, but there was no correlation between SR and other factors. Findings and Conclusions: Elasticity in MCLs of baseball players decreases with age, and increases on the throwing side. Quantifying the elasticity of the elbow MCL in this study will facilitate future investigation of how ligament characteristics could affect MCL injuries.
\end{abstract}

Key words : medial collateral ligament, elbow, elastography

\section{Introduction}

Valgus force is repeatedly exerted on the elbow joint during the throwing motion of a baseball player, and tensile stress is thus placed on the MCL, possibly resulting in injury. MCL injuries are generally diagnosed using stress X-rays, magnetic resonance imaging (MRI), or ultrasonography (US). However, while these methods have proven useful in the diagnosis of MCL injury ${ }^{1-3)}$, the evaluation methods are difficult, with other reports of the extent of abnormal findings on MRI or instability in stress X-rays not correlating with the return rate to competition ${ }^{4)}$. Furthermore, there seems to be no correlation between ultrasound-based elbow instability on one side, and elbow pain, pitching injury, or self-evaluation on the other ${ }^{5)}$,

\footnotetext{
1) Department of Orthopaedic Surgery, Showa University School of Medicine, 1-5-8 Hatanodai, Shinagawa-ku, Tokyo 142-8666, Japan.

2) Department of Orthopaedic Surgery, Waseikai Oowaki Hospital.

3) Department of Orthopaedic Surgery, Showa University Toyosu Hospital.

* To whom corresponding should be addressed.
} 
which makes it difficult to evaluate the method. On the other hand, ultrasonography is being used routinely in orthopedic disease diagnosis such as ankle ligament injuries and rotator cuff injuries due to its convenience for dynamic evaluation and its noninvasive nature. Real-time tissue elastography is a technique for imaging, in real-time, the relative hardness of tissue using ultrasonic diagnostic equipment; it is clinically applied in therapeutic areas of the mammary gland $^{6)}$, thyroid diseases ${ }^{7)}$, and liver deseases ${ }^{8)}$. By evaluating elasticity in the elbow MCL in real time, practitioners might be able to compensate for the parts that are difficult to evaluate using stress X-rays or MRI examinations alone. Therefore, we sought to establish a control group for future evaluation of MCL injuries by measuring the elasticity of normal elbow MCLs.

\section{Materials and methods}

We evaluated 72 elbows in 36 male baseball pitchers with no history of pitching injuries (defined as subjects with no pain that would keep them from competition at the time of the study). These included 12 junior high students, 12 high school students, and 12 university students, with a mean age of 17 (age range 12-22 years). The US system equipment included HI VISION Avius (Hitachi Aloka Medical, Mitaka, Tokyo) machines equipped with EUP-L65 high-resolution 6.0-14.0-MHz linear-array probes, an EZU-TECPL1 acoustic coupler attached to the probes for quantification, and real-time tissue elastography. We used an acoustic coupler because there was not enough fixed-hardness tissue such as fat around the MCLs to compare hardness. We measured the subject in a position such that stress was applied by the forearm's own gravity on the MCL, as per the gravity test, with the subject's forearm in supination, elbow joint at $30^{\circ}$ of flexion, and shoulder joint at $90^{\circ}$ abduction with the subject in a supine position. Elastography measurements were conducted by manually applying pressure so as to maintain the appropriate compression depth and interval between the pressure applications while referring to a strain graph.

For evaluation, we used the strain ratio (SR) calculated as the ratio of the strain (in other words, the minimal change resulting from each compression) on the MCL to that of the acoustic coupler, i.e., the coupler strain measurement divided by the compression-induced strain. The measurement site was determined to be $1 \mathrm{~cm}$ from the midpoint of the attachment site of the medial epicondyle of the humerus on the anterior oblique ligament (AOL) of the MCL, with measurements conducted while applying pressure longitudinally along the ligament. In ultrasound elastography imaging, the color display changes to red as the strain in the tissue increases with pressure, and shifts from yellow to green to blue as the strain decreases (Fig. 1). We conducted measurements by applying pressure as described above. A measurement was conducted once for each elbow per subject, and a representative value was calculated as the mean value of three median values measured at five selected locations in each elbow that met the appropriate conditions based on the recorded video.

We analyzed the SR correlations with age, throwing and non-throwing side, history of elbow injuries, state of the elbow joint, and state of the shoulder joint. Thus, we examined the correlation between SR and (if any) history of visits to the hospital for elbow pain due to 
(A) (B)

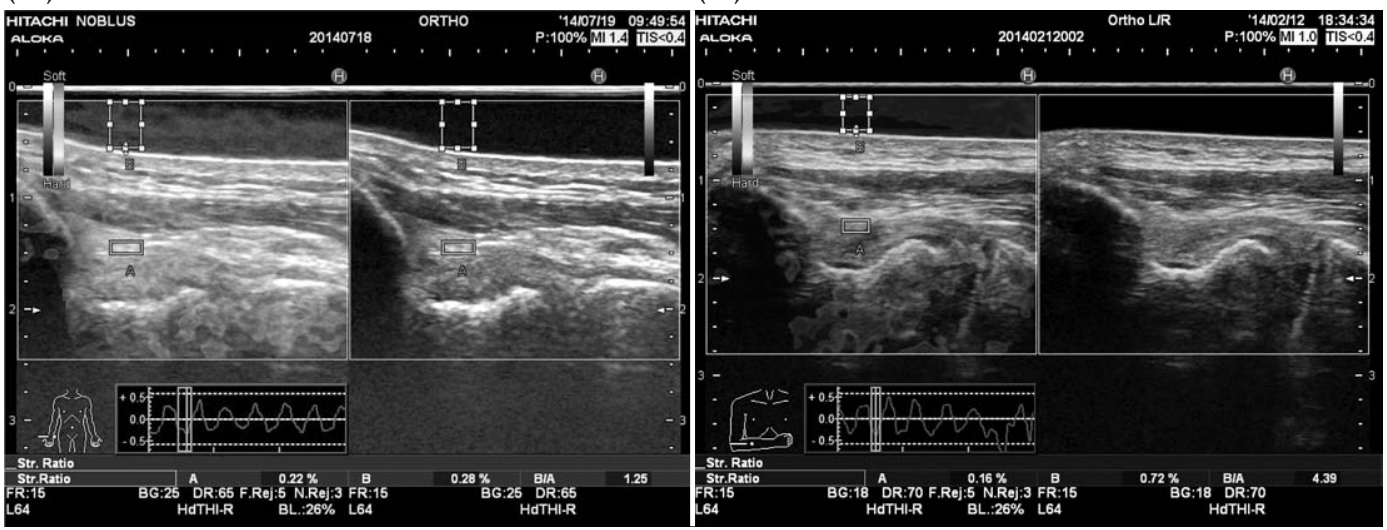

Fig. 1. Color display of the elastography and ROI setting (the range in MCL and coupler indicated in the square box) for measuring SR. Example display of lower SR (A) and higher SR (B)

injury. Furthermore, the SR correlations were examined with respect to MCL tenderness, the cubital tunnel Tinel's sign, the milking test, the moving valgus stress test for the state of the elbow joint, and omalgia for the state of the shoulder joint.

All statistical analyses were performed using software (JSTAT 9.3 for Windows). Statistical analyses were conducted using the paired t-test, the Mann-Whitney U-test, and one-way analysis of variance.

\section{Results}

In all cases, imaging revealed a mixed pattern in the elbow MCL from yellowish-green to blue (Fig. 1).

1) Age and $S R$

We found a correlation between age and SR on the throwing side and non-throwing side, with significantly higher values in SR as age increases (throwing side $P=0.011$, non-throwing side $P=0.041$ ) (Fig. 2). The mean for SR on the throwing side for the junior high school student group, high school student group, and university student group was 1.18, 2.33, and 2.72, respectively, and on the non-throwing side it was $1.81,2.67$, and 3.08, respectively (Fig. 3). The junior high group exhibited significantly lower SR values compared to the high school and university student groups on both throwing and non-throwing sides $(P<0.05)$.

2) Throwing side SR and non-throwing side SR

The mean SR was 2.07 for the throwing side and 2.52 for the non-throwing side, and the throwing side exhibited significantly lower SR values compared to the non-throwing side $(P=$ $0.034)$.

3) History of elbow injury on the throwing side, and SR

In our study group, 11 subjects had a history of elbow injury on the throwing side (consultation history for elbow pain), while 25 did not, and no significant difference between them was found on the throwing side SR $(P=0.823)$ (Fig. 4). 
(A)

\section{Age and SR ( throwing side)}

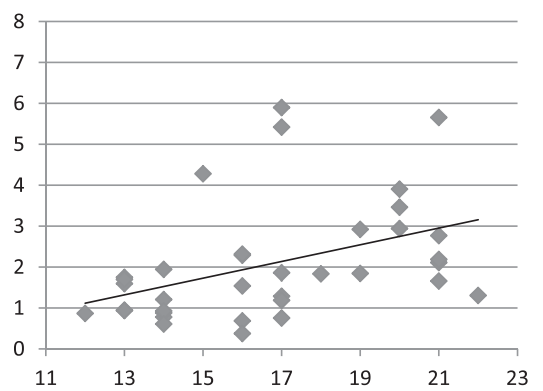

(B)

\section{Age and SR (non-throwing side)}

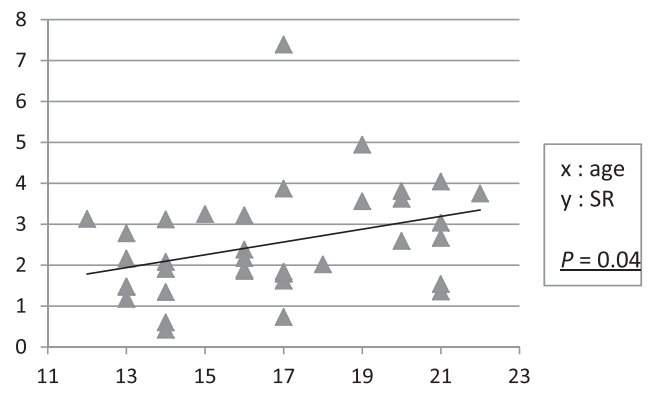

Fig. 2. Scatter diagram of age and SR. We found a correlation between age and SR on both the throwing side and non-throwing side. SR values increased with age on both sides.
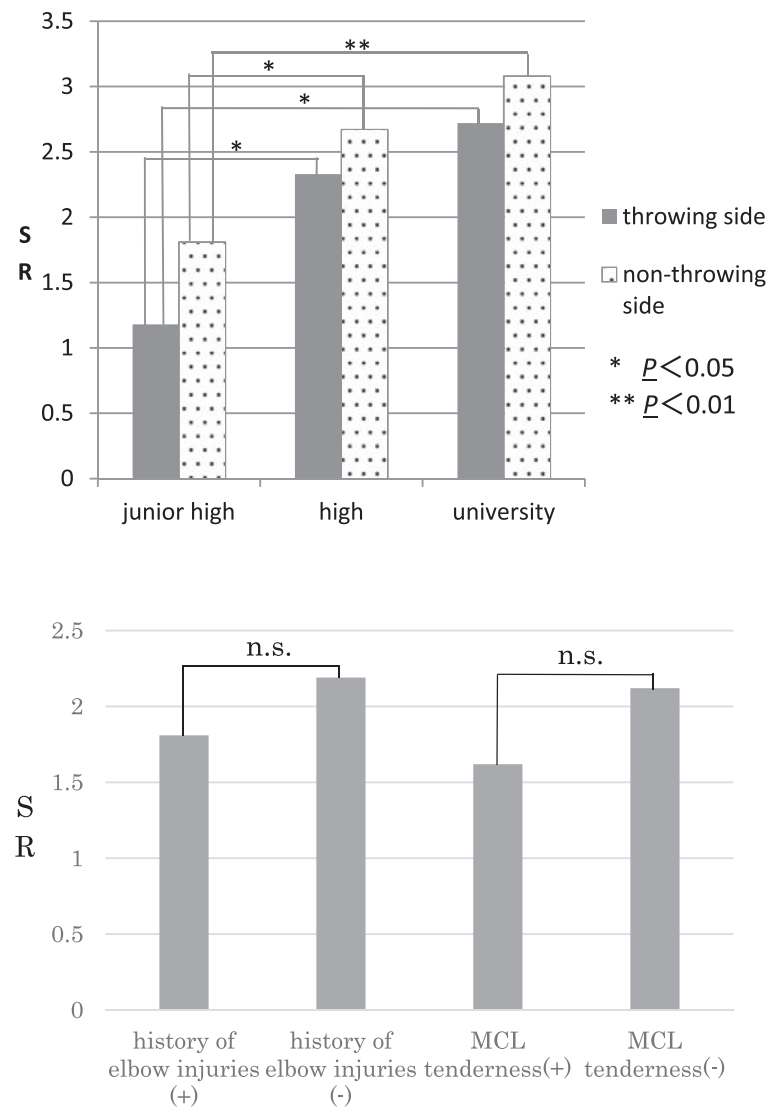

Fig. 3. We found a significant difference in SR between the junior high school student group and high school student group, and the junior high school student group and university student group on both sides. The junior high school student group exhibited lower SR values compared to the other groups on both sides.

Fig. 4. There was no significant difference between throwing side SR and the history of elbow injuries or MCL tenderness

4) Status of throwing side elbow joint and SR

We found no significant difference in SR between any of the positive groups and respective negative groups: MCL tenderness was present in 3 subjects and absent in 33 subjects $(P=$ 0.829); and cubital tunnel Tinel's sign was present in 3 subjects and absent in 33 subjects ( $P$ 
$=0.957)$. The milking test produced 3 positive subjects and 33 negative $(P=0.088)$, while 1 subject tested positive for the moving valgus stress test and 35 subjects tested negative. While there was a trend for high SR values in the group that tested positive in the milking test, the differences were not significant.

5) Status of throwing side shoulder joint and SR

Omalgia was present in 6 subjects and absent in 30 subjects. No significant difference in SR was found between the positive group and negative group for omalgia $(P=0.725)$.

\section{Discussion}

Surgical indications and techniques for managing elbow collateral ligament insufficiency in throwing athletes are continuing to evolve. Essentially, the lateral collateral ligament must be surgically repaired, whereas the MCL will heal in some instances, although repair is needed in athletes who place high valgus stress on the elbow joint such as during throwing. Morrey et $a l^{9)}$ defined the MCL as the primary constraint of the elbow joint to valgus stress and the radial head as a secondary constraint, while Floris et al ${ }^{10)}$ defined the AOL as the primary constraint to valgus and internal rotatory forces. Clinicians should consider reconstruction of the injured MCL in those patients who are unable to return to sports activities or work after an interval of rest and rehabilitation ${ }^{11)}$. On the radiological diagnosis of MCL injury, Fritz and Steinbach ${ }^{1)}$ reported on the usefulness of MRI for diagnosing elbow joint disease including MCL injury, while Lee et $a l^{2)}$ proposed comparing uninjured and injured populations in reporting the usefulness of stress X-rays. Rijke et $a l^{3)}$ reported on the usefulness of stress X-rays for accurate diagnosis of large and complete tears and for distinguishing between such tears or partial tears and normal tears. However, Watanabe et $a l^{4)}$ reported that a return to sports did not necessarily correlate with either the extent of instability in stress X-rays or abnormal findings on MRI after conservative therapy for elbow MCL injuries in baseball players ${ }^{4)}$. Furthermore, such results are difficult to assess with repeated periodic evaluations using these tests in terms of time. On the other hand, ultrasonography allows for real-time, dynamic evaluation; it can be easily performed in an outpatient clinic as the physician provides an explanation of the procedure to the patient. Ferrara $e t a l^{12)}$ reported the efficiency of ultrasound to obtain precise information on the structure of the MCL, while Harada et $a l^{5)}$ found no correlation between self-evaluation and pitching difficulty, or elbow pain and instability by ultrasound.

In this study, therefore, we focused on elastography for evaluating tissue elasticity by ultrasonography in young baseball pitchers. Elastography is already clinically applied in mammary gland-related fields, having become a supplementary diagnostic tool for breast cancer in evaluating the Tsukuba Elasticity Score and FLR (fat-lesion ratio) for strain in target tissue and fat ${ }^{13)}$. Elastography-based examination is also conducted in orthopedic fields. Tsujimura ${ }^{14)}$ performed a temporal evaluation of gastrocnemius contusion, for advocating the usefulness of elastography as an indicator for return to sport and for monitoring the recovery process, while Kijima et $a l^{15)}$ compared elasticity of the rotator cuff and coracoacromial ligament using elastography. Tanaka et al ${ }^{16)}$ compared the flexor digitorum superficialis muscle and median 
nerve elasticity in healthy subjects. To the best of our knowledge, elastography has not been evaluated for the measurement of elbow MCL elasticity.

In the present study, SR increased (meaning elasticity decreased) with age. Interestingly, Ichihashi $e t a l^{17)}$ reported a decrease in both the elastic fiber and moisture content of human tissues with increasing age, causing loss of elasticity as insoluble collagen increases in the skin. We presumed therefore that SR increases with age, and in this study the throwing side exhibited significantly lower SR values compared to the non-throwing side. In support of this, Nazarian et $a l^{18)}$ reported elbow joint laxity with valgus stress in the throwing side of professional baseball pitchers, and Sasaki et $a l^{19)}$ found medial elbow laxity and an increase in the elbow valgus on the throwing side of baseball players compared with nonplayers. We thus presumed that the MCL enlarges and loosens by repeated extension stress to the throwing side.

There are several issues to consider with elastography-based measurements including technical points such as reproducibility and proficiency level when conducting ultrasonography and elastography. Namely, compression must be repeatedly applied while maintaining the optimal compression depth, initial pressure, and pressure cycle as well as accurately visualizing the AOL of MCL in the elbow. During this procedure, the position of the probe must also remain still. Hence discrepancies in measurement values may arise when multiple examiners with varying levels of skill are involved, or when an inappropriate location is chosen from the recorded video; in the latter case, experience in image selection may also be necessary. To check intrarater reliability, we measured SR 8 times for 3 subjects, and then measured it again 3 days later. Intra-rater reliability showed an intraclass correlation coefficient of 0.78 .

Future studies should investigate the usefulness of elastography-based elasticity evaluation as an indicator for a return to competition and in the screening and evaluating the severity of elbow MCL injuries by comparing between an elbow MCL injury group and the results obtained in the present study as the control group.

In conclusion, comparative studies of elbow MCL elasticity in junior high school, high school, and university student baseball players who were free from pitching injury using ultrasound elastography, revealed that elbow MCL elasticity was increased in the throwing side, and decreased with increasing age. As a result, we were able to establish a control group necessary to investigate the usefulness of elastography-based elasticity evaluation in a group of subjects with elbow possible MCL injuries in the future.

\section{Conflict of interest}

The authors have declared no conflict of interest.

\section{References}

1) Fritz RC, Steinbach LS. Magnetic resonance imaging of the musculoskeletal system. Part 3. The elbow. Clin Orthop Relat Res. 1996;324:321-339.

2) Lee GA, Katz SD, Lazarus MD. Elbow valgus stress radiography in an uninjured population. Am J Sports Med. 1998;26:425-427. 
3) Rijke AM, Goitz HT, McCue FC, et al. Stress radiography of the medial elbow ligaments. Radiology. 1994;191:213216.

4) Watanabe M, Yonekawa S, Hattori M, et al. Sports comebacks after conservative therapy for elbow MCL injury in baseball players. Japanese Journal of Orthopaedic Sports Medicine. 2012;32:271-275. (in Japanese).

5) Harada M, Takahara M, Suzuki T, et al. Elbow injuries in high school baseball players. Japanese Journal of Clinical Sports Medicine. 2010;18:442-447. (in Japanese).

6) Hashimoto H. Evaluation of breast tumors with real-time tissue elastography in differential diagnosis. $M E D I X$. 2004;41:11-14. (in Japanese).

7) Tanaka K, Fukunari N, Ito K, et al. Clinical evaluation of thyroid tumor with real-time tissue elastography. MEDIX. 2004;41:7-10. (in Japanese).

8) Jeong WK, Lim HK, Lee HK, et al. Principles and clinical application of ultrasound elastography for diffuse liver disease. Ultrasonography. 2014;33:149-160

9) Morrey BF, Tanaka S, An KN. Valgus stability of the elbow. A definition of primary and secondary constraints. Clin Orthop Relat Res. 1991;265:187-195.

10) Floris S, Olsen BS, Dalstra M, et al. The medial collateral ligament of the elbow joint, anatomy and kinematics. $J$ Shoulder Elbow Surg. 1998;7:345-351.

11) Williams RJ 3rd, Urquhart ER, Altchek DW. Medial collateral ligament tears in the throwing athlete. Instr Course Lect. 2004;53:579-586.

12) Ferrara MA, Marcelis S, Popovic N, et al. Modifications of the elbow induced by the practice of handball on radiography, US and MRI. JBR-BTR. 1999;82:222-227.

13) Japan Association of Breast and Thyroid Sonology ed. : Breast ultrasonography guidelines. 2nd ed. Tokyo. Nankodo;2008:133-137. (in Japanese).

14) Tsujimura T. Ultrasonography for pulled muscles: The use of elastography (tissue elasticity imaging). The Journal of the Japanese Society of Orthopedic Ultrasonics. 2007;18:19-22. (in Japanese).

15) Kijima Y, Minakawa $\mathrm{H}$, Tomioka $\mathrm{T}$, et al. Elasticity of the coracoacromial ligament in the in vivo shoulder: Measurements using ultrasound elastography. The Shoulder Joint. 2008;32:357-360. (in Japanese).

16) Tanaka T, Ogawa T, Yoshii Y. Elastography-based evaluation of the median nerve in healthy subjects. The Journal of Japanese Society for Surgery of the Hand. 2012;28:423-425. (in Japanese).

17) Ichihashi M, Yagi M, Nomoto K, et al. Glycation stress and photo-aging in skin. Anti-Aging Medicine. 2011;8:323329.

18) Nazarian LN, McShane JM, Ciccotti MG, et al. Dynamic US of the anterior band of the ulnar collateral ligament of the elbow in asymptomatic major league baseball pitchers. Radiology. 2003;227:149-154.

19) Sasaki J, Takahara M, Ogino $\mathrm{T}$, et al. Ultrasonographic assessment of the ulnar collateral ligament and medial elbow laxity in college baseball players. J Bone Joint Surg Am. 2002;84-A:525-531.

[Received December 4, 2014 : Accepted December 26, 2014] 